\title{
Cyber-Physical Disaster Response of Power Supply Using a Centralised-to-Distributed Framework
}

\author{
Pudong Ge \\ Electrical and Electronic Engineering \\ Imperial College London \\ London, U.K. \\ pudong.ge19@imperial.ac.uk
}

\author{
Charalambos Konstantinou \\ CEMSE Division \\ KAUST \\ Thuwal, Saudi Arabia \\ charalambos.konstantinou@kaust.edu.sa
}

\author{
Fei Teng \\ Electrical and Electronic Engineering \\ Imperial College London \\ London, U.K. \\ f.teng@imperial.ac.uk
}

\begin{abstract}
This paper proposes a cyber-physical cooperative recovery framework to maintain critical power supply, enhancing power systems resilience under extreme events such as earthquakes and hurricanes. Extreme events can possibly damage critical infrastructure in terms of power supply, on both cyber and physical layers. Microgrid (MG) has been widely recognised as the physical-side response to such blackouts, however, the recovery of cyber side is yet fully investigated, especially the cooperatively recovery of cyber-physical power supply. Therefore, a centralised-to-distributed resilient control framework is designed to maintain the power supply of critical loads. In such resilient control, controller-to-controller (C2C) wireless network is utilised to form the emergency distributed communication without a centralised base station. Owing to the limited reliable bandwidth that can be employed in $\mathrm{C2C}$ networks, the inevitable delay is considered in designing a discrete control framework, and the corresponding stability criteria are given quantitatively. Finally, the cyber-physical recovery framework is demonstrated effectively through simulations in MATLAB/Simulink.
\end{abstract}

Index Terms-Disaster response, resilience, microgrids, centralised-to-distributed, wireless communications.

\section{INTRODUCTION}

Recently, renewable energy resources have an increasingly trend of dominating the power supply especially in distributed power networks [1], [2]. At the same time, the resilient requirement of power supply has also been widely raised after extreme weather events started to occur more frequently around the world [3], [4]. Such events often lead to blackouts during which the main grid becomes unavailable. Using renewable distributed generators (DGs) to provide emergency power supply for critical loads such as hospitals and critical factories is a widely recognised solution (a concept of MG) to enhance the resilience of power supply [2], [5]. For example, the Consortium for Electric Reliability Technology Solutions (CERTS)-enabled MG maintained power, water, heat of Brevoort building in Greenwich Village, NY, USA, during the week of wide spread utility outages due to Hurricane Sandy in late 2012 [6]. In Japan, Sendai MG and Roppongi Hills MG, functioned as secure islands following the earthquake and tsunami, demonstrated that well-developed localized en-

This work was supported by EPSRC under Grant EP/T021780/1 and by The Royal Society under Grant RGS/R1/211256. Corresponding author: Dr Fei Teng(f.teng@imperial.ac.uk)). ergy systems are the key to maintaining critical emergency services [7].

Currently, the research on MG providing post-event power supply mostly focuses on the control strategy design during the post-event period. The islanded MG control strategy has been widely investigated to guarantee the frequency and voltage stability in a small scale [8]-[11], which focus on the power supply recovery from a physical perspective, especially using the grid-forming concept [12]. Recently, scholars started to consider MG control from the perspective of the cyberphysical system under extreme conditions. Ad-hoc communication technology has been utilised to realise the self-organised MGs in response to disasters [13]. The concept of dynamic MGs, including the configurable cyber and physical layers, has been adopted to enable the autonomous operation of distribution systems [14].

Despite the recent adoption of dynamic MGs, the cyberphysical collaborative response strategy during the pre-event and post-event periods, especially the cooperative design of communication network and control strategy, has not been comprehensively examined in literature. In this paper, we design a centralised-to-distributed resilient control framework of distributed power networks to maintain the critical power supply by utilising MG clusters isolated from the distribution system, thus enhancing the system resilience. Under such centralised-to-distributed transition, the communication network is transited from base station supporting mode under normal operating conditions to controller-to-controller mode under extreme conditions. The controller-to-controller communication only requires one wireless module located at the local controller, which is originally necessary for receiving the instructions in the pre-event normal condition. Moreover, in order to guarantee reliable control, the bandwidth available for the post-event extreme condition is limited when using private wireless network specific for power industry [15]. To summarise, the contributions of the paper are listed as follow:

1) A centralised-to-distributed resilient control framework is proposed from the cyber-physical perspective, where the post-event response is designed in a low-cost communication solution.

2) A resource allocation based controller-to-controller distributed communication provides the optimal cyber so- 


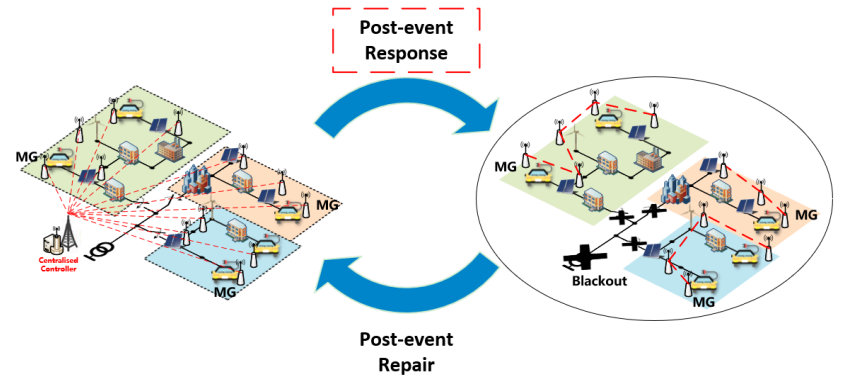

Fig. 1. Centralised-to-distributed resilient control framework.

lution for post-event response using private wireless network. Under such emergency wireless network, a discrete control system is designed fully considering the communication delay derived from the limited bandwidth.

The remainder of this paper is organised as follows: Section II introduces the framework and detailed system models, while Section III provides the cyber-physical system co-design method of the resilient control framework. In Section IV, simulation results are given, and Section $\mathrm{V}$ concludes the paper.

\section{Cyber-Physical POWER Supply Recovery FRAMEWORK AND SYSTEM MODEL}

\section{A. Framework of Cyber-Physical Power Supply Recovery}

A centralised-to-distributed resilient control framework, as shown in Fig. 1, is designed in response to blackout events caused by disasters. In the pre-event normal condition, the distributed power network is regulated by the centralised controller to maintain optimal performance. At the same time, the centralised controller arranges the post-event planning for local controllers of DGs in the cyber and physical layers. Given that the disaster can lead to damage of cyber-physical devices (e.g., base station or power lines), the critical power supply transits from pre-event centralised mode to post-event distributed mode due to the unavailability of centralised power supply and communication network.

During the post-event period, MG clusters maintain the critical power supply using the emergency distributed C2C wireless communication network, the communication plan of which has been scheduled in advance during normal operation. Then, after the repair process, the power supply returns to the normal condition. Such centralised-to-distributed resilient control framework operates in period cycles to guarantee the total availability of critical power supply even after extreme events. It should be noted that extreme events such as cyberattacks can also be mitigated through the proposed centralisedto-distributed framework. Once cyber-attacks, which possibly make the centralised control system unreliable, are detected by active defense strategies [16], such framework is able to enhance cyber resilience.

The core issue for the post-event response is the scheduling of cyber-physical emergency MG solution depicted in Fig. 2.
In the following, both cyber and physical models are introduced, and the corresponding problems are formulated.

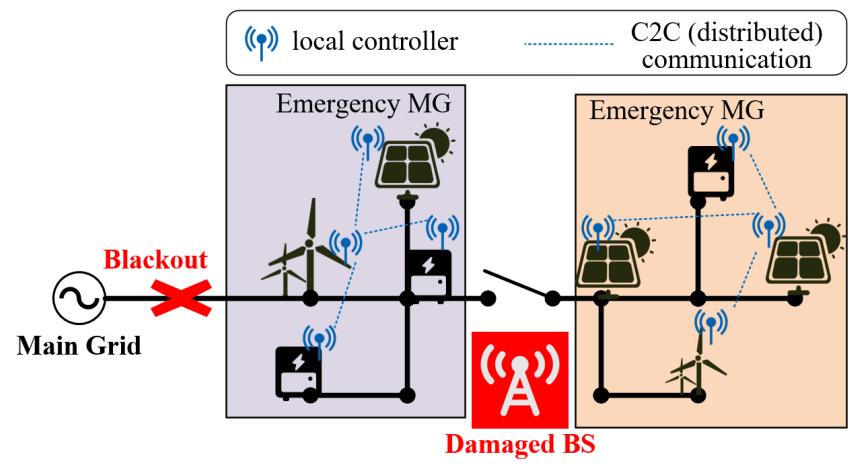

Fig. 2. Cyber-physical emergency MG during post-event periods.

\section{B. Physical-Layer Model}

Emergency MG for physical response consists of different categories of distributed energy resources (DERs), which can be generally modelled as inverter-based DGs. For an emergency MG, the modelling focuses on the generators only because the details of temporarily operating MG are not realistic. Thus, models focus on the inverter-based DG itself by appropriate approximation. The output active power of DG $i$ can be expressed as the following with a line impedance $Z_{i j}=R_{i j}+j X_{i j}$ between DG $i$ and DG $j$ : [17]

$$
P_{i}=P_{i, \text { Load }}+\sum_{j=1}^{N_{i}} \frac{V_{i} V_{j}}{X_{i j}} \sin \left(\theta_{i}-\theta_{j}\right)
$$

where $N_{i}$ denotes the number of DGs physically connected to DG $i, P_{i, \text { Load }}$ is the active power demand of the load at bus $i$, and $V_{i}$ and $\theta_{i}$ are the bus voltage and the angle at bus $i$, respectively. Due to the fact that the phase difference $\left(\theta_{i}-\theta_{j}\right)$ is small [18], $\sin \left(\theta_{i}-\theta_{j}\right) \approx\left(\theta_{i}-\theta_{j}\right)$, which means the active power can be controlled by the difference of phase angle. Thus, the droop-based power control loop can be formulated as [19]:

$$
\omega_{i}=\omega_{n i}-m_{P i} P_{i}
$$

where $\omega_{n i}$ is the nominal set point for frequency regulation, $\omega_{i}$ is the angular frequency of the $i_{t h}$ generator, $m_{P i}$ and $P_{i}$ are respectively frequency droop coefficient and active power output of the $i_{t h}$ generator.

As depicted in Fig. 3, each DG unit contains a DC/AC inverter, an inductor-capacitor (LC) filter, and a resistorinductor connection locally. Its primary controller is composed of power controller and inner controller. The primary control through Eq. (2) cannot eliminate the frequency deviations from the reference without the coordination of DGs, and the secondary control is employed to achieve frequency regulation and accurate active power sharing, i.e.,

$$
\begin{aligned}
& \lim _{t \rightarrow \infty} \omega_{i}=\omega_{\text {ref }} \\
& \lim _{t \rightarrow \infty}\left|\frac{P_{i}}{P_{\max , i}}-\frac{P_{j}}{P_{\max , j}}\right|=0
\end{aligned}
$$




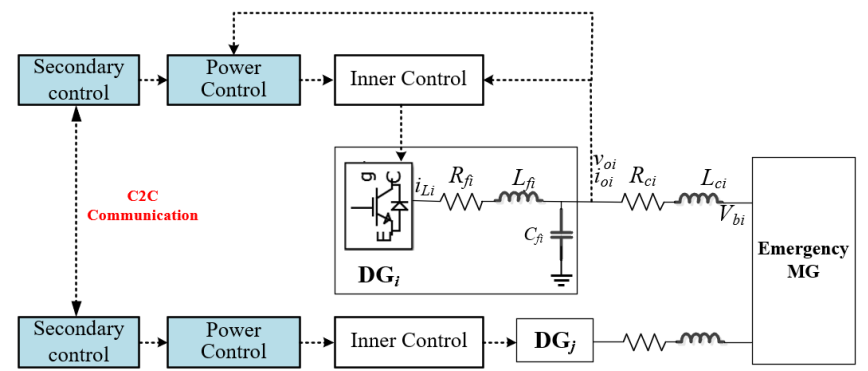

Fig. 3. Block diagram of an inverter-based MG.

where $P_{\max , i}$ denotes the active power ratings of the $i_{t h}$ generator, and Eq. (4) is equivalent to $\lim _{t \rightarrow \infty}\left|m_{P i} P_{i}-m_{P j} P_{j}\right|=$ 0 by approximately setting $m_{P i} P_{\max , i}=m_{P j} P_{\max , j}$.

\section{Cyber-Layer Model}

The DGs in the emergency MG require information sharing to reach the control objectives of Eqs. (3) and (4). As described in Fig. 2 and Fig. 3, the C2C communication is utilised to form the distributed communication framework. Such distributed communication can be modelled by a graph $\mathcal{G}=\{\mathcal{V}, \mathcal{E}, \mathcal{A}\}$ : the node set $\mathcal{V}$ represents DGs, the edge set $\mathcal{E}=\mathcal{V} \times \mathcal{V}$ represents the available communication links among the cyber layer, and adjacent matrix $\mathcal{A}=\left[a_{i j}\right] \in \mathbb{R}^{N \times N}$ denotes the relationship in the communication network. More specifically, $a_{i i}=0$ and $a_{i j}=1, i \neq j$ only if $\left(v_{i}, v_{j}\right) \in \mathcal{E}$, otherwise $a_{i j}=0$. For $\mathcal{G}$, the out-degree matrix is denoted by $\mathcal{D}=\operatorname{diag}\left\{d_{i}\right\}, d_{i}=\sum_{v_{j} \in N_{i}} a_{i j}$, and the Laplacian matrix $\mathcal{L}=\mathcal{D}-\mathcal{A}$ [20]. In the control framework, we consider the distributed $\mathrm{C} 2 \mathrm{C}$ communication network as an undirected graph that needs to be connected to realize consensus control, and such data exchange between DGs occurs in a timetriggered manner.

\section{Research Questions}

With regards to Eq. (2) and the control objectives of Eqs. (3) and (4), two problems need to be solve cooperatively:

1) How to design a $\mathrm{C} 2 \mathrm{C}$ distributed communication network under low-bandwidth backup communication resource to achieve the minimum delay of the data exchange?

2) How to design a control system to maintain stability and reach objectives given the delay in the communication network?

\section{Co-Design OF CYBER-PhysiCAL EMERGENCY MICROGRID}

In this section, the problems described in Section II-D are mitigated through cyber-physical consideration co-design. Firstly, the time delay existing in the $\mathrm{C} 2 \mathrm{C}$ communication network is quantitatively determined by the resource allocation optimisation of wireless communication. Then, a distributed control framework is developed for the delay-dependent consensus control. Both design processes are dynamically updated in the pre-event stage to be ready for use in the post-event stage.

\section{A. Pre-designed Resource Allocation of C2C Wireless Com- munication}

Assume the wireless network for distributed communication of emergency MG containing $N$ DGs, and the wireless connection is described by $\mathcal{A}$, and $a_{i j}=1$ represents that there exists a $\mathrm{C} 2 \mathrm{C}$ pair. $\mathrm{C} 2 \mathrm{C}$ pairs in the set $\mathcal{E}=\{1,2, \ldots, E\}$ employ the total bandwidth for reliable communication in the emergency state. The total bandwidth is divided into $S$ subcarriers in the set $\mathcal{S}=\{1,2, \ldots, S\}$. The channel gain and the transmit power for the $\mathrm{C} 2 \mathrm{C}$ pair $e$ on the sub-carrier $s$ are represented by $g_{e, s}$ and $p_{e, s}$ respectively. Then, the signal-tonoise ratio (SNR) for the $\mathrm{C} 2 \mathrm{C}$ pair $e$ on the sub-carrier $s$ [21], can obtained by:

$$
\gamma_{e, s}=\frac{p_{e, s} g_{e, s}}{\sigma^{2}}
$$

where $\sigma^{2}$ denotes the noise power in the channel. The reachable instantaneous $\mathrm{C} 2 \mathrm{C}$ transmission rates in bits per second are computed through Shannon formula as:

$$
R_{e}=w \sum_{s=1}^{S} \alpha_{e, s} \log _{2}\left(1+\gamma_{e, s}\right)
$$

where $\alpha_{e, s}$ is a binary variable to determine if sub-carrier $s$ is assigned to the $\mathrm{C} 2 \mathrm{C}$ pair $e$, and $w$ denotes the bandwidth of one sub-carrier.

In the emergency wireless network, normal QoS (Qualityof-Service) requirement is discarded, and it is considered as the objective function in the resource allocation optimisation. The optimisation aims to maximize transmission rate defined in Eq. (6), so as to minimize the delay existing in the $\mathrm{C} 2 \mathrm{C}$ distributed wireless communication network. Based on the analysis above, the optimisation problem can be formulated as follows:

$$
\begin{aligned}
& \operatorname{maximize} \min \left(R_{e}\right) \\
& \text { subject to : } \\
& \mathrm{C}_{1}: \quad \sum_{e=1}^{E} \alpha_{e, s} \leq 1, \forall s \in \mathcal{S} \\
& \mathrm{C}_{2}: \quad \alpha_{e, s} \in\{0,1\}, \forall e, s \\
& \mathrm{C}_{3}: \quad p_{\mathrm{cst}}+\sum_{e \in \mathcal{E}_{i}} \sum_{s=1}^{S} p_{e, s} \leq p_{\max , i}
\end{aligned}
$$

where Eqs. $(7 b)-(7 c)$ are the exclusive sub-carrier allocation in the communication links. Eq. (7d) represents each communication unit in the controller should satisfy the maximum power consumption requirement. In the constraint $\mathrm{C}_{3}$, the set $\mathcal{E}_{i}$ denotes the edges that DG $i$ shares information with neighbours, while $p_{\text {cst }}$ is the constant circuit power consumed by wireless communication module of controller installed for DG $i$. The whole resource allocation optimisation for emergency wireless network can be formulated as a mixed integer nonlinear optimisation programming (MINLP), which 
TABLE I

Distributed CONTROL FrAMEWORK FOR EMERGENCY MGS

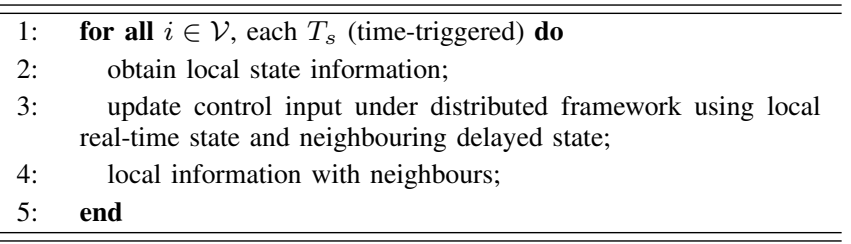

can be directly solved by MATLAB/YALMIP using MOSEK [22], [23].

The MG clusters are formed naturally and determined by the switches that are installed to enable such cyber-physical resilient control framework. As shown in Eqs. (5) and (6), the data rate is positively related to the channel gain, and the channel gain is negatively related to wireless transmitting distance. Hence, an efficient way to form the wireless network is to connect DGs to share information with its nearest neighbours. Due to the designed framework where the postevent response is scheduled during the pre-event period and the location of DGs are known, the wireless communication network for each MG can be formed naturally with $E=N-1$, which can satisfied the connectivity requirements discussed in Section II-C.

After solving the optimisation problem Eqs. (7a) - (7d), we can obtain the maximized data rate $R_{\min }=\min R_{e}^{*}$, thus the delay can be calculated as:

$$
\tau=\frac{L_{\text {packet }}}{R_{\min }}
$$

where $L_{\text {packet }}$ denotes the packet size for the $\mathrm{C} 2 \mathrm{C}$ distributed control.

\section{B. Delay-Dependent C2C Distributed Control}

For an emergency response, the wireless $\mathrm{C} 2 \mathrm{C}$ communication normally has limited bandwidth and each controller hardware has limited transmit power, thus the control cannot be supported in a high-frequency way, thus we design the distributed control framework with sampling interval $T_{s}>\tau$ as shown in Table I. Owing to the relatively low-frequency time-triggered control framework, we model the dynamics of the system Eq. (2) in a discrete manner:

$$
\begin{aligned}
\omega_{n i}(k+1) & =\omega_{i}(k+1)+m_{P i} P_{i}(k+1) \\
& =\omega_{i}(k)+u_{\omega i}(k)+m_{P i} P_{i}(k)+u_{P i}(k)
\end{aligned}
$$

from which we describe the frequency and active power dynamics with:

$$
\left\{\begin{array}{r}
\boldsymbol{x}_{\omega}(k+1)=\boldsymbol{x}_{\omega}(k)+\boldsymbol{u}_{\omega}(k) \\
\boldsymbol{x}_{P}(k+1)=\boldsymbol{x}_{P}(k)+\boldsymbol{u}_{P}(k)
\end{array}\right.
$$

where $\boldsymbol{x}_{\omega}=\left[x_{\omega 1}, \ldots, x_{\omega n}\right]^{T}=\left[\omega_{1}, \ldots, \omega_{n}\right]^{T}$, $\boldsymbol{x}_{P}=\left[x_{P 1}, \ldots, x_{P n}\right]^{T}=\left[m_{P 1} P_{1}, \ldots, m_{P n} P_{n}\right]^{T}$, $\boldsymbol{u}_{\omega}=\left[u_{\omega 1}, \ldots, u_{\omega n}\right]^{T}$, and $\boldsymbol{u}_{P}=\left[u_{P 1}, \ldots, u_{P n}\right]^{T}$.

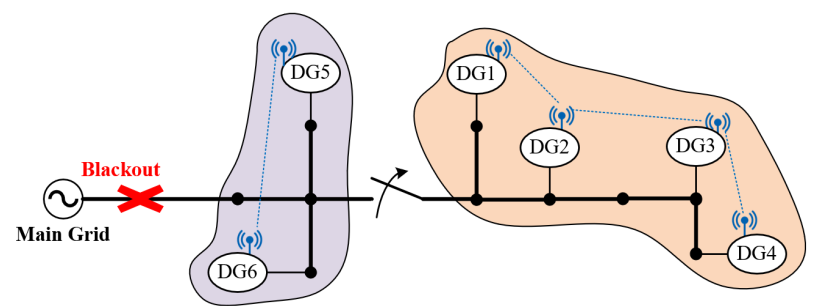

Fig. 4. Diagram of the distributed power network topology for testing disaster response.

The distributed averaging proportional integral (DAPI) discrete controller is formulated by utilising extensively studied consensus protocol [24]:

$$
\left\{\begin{array}{l}
u_{\omega i}(k)=K_{\omega}\left(x_{\omega, r e f}-x_{\omega i}(k)\right) \\
u_{P i}(k)=K_{P} \sum_{j \in N_{i}} a_{i j}\left(x_{P j}(k)-x_{P i}(k)\right)
\end{array}\right.
$$

where $K_{\omega}, K_{P}>0$ are the designed control gains. However, as describe in Table I, thanks to the design of $T_{s}>\tau$, the delay caused by low-bandwidth wireless communication is an one time step. Then, by defining $\boldsymbol{x}_{\omega}^{e}=\boldsymbol{x}_{\omega}-x_{\omega, \text { ref }} \mathbf{1}_{N}$, where $\mathbf{1}_{N}$ denotes a column vector with all elements being ones, the system dynamics can be expressed in a matrix form:

$$
\left\{\begin{array}{l}
\boldsymbol{x}_{\omega}^{e}(k+1)=\left(1-K_{\omega}\right) \boldsymbol{x}_{\omega}^{e}(k) \\
\boldsymbol{x}_{P}(k+1)=\boldsymbol{x}_{P}(k)-K_{P} \mathcal{D} \boldsymbol{x}_{P}(k)+K_{P} \mathcal{A} \boldsymbol{x}_{P}(k-1)
\end{array}\right.
$$

Theorem 1: For the MG controlled under Table I and Eq. (11) with an undirected graph $\mathcal{G}$ modelled in a distributed communication structure, the distributed frequency restoration and active power sharing can be achieved as Eqs. (3) and (4) asymptotically if the conditions:

$$
0<K_{\omega}<2 \quad \text { and } \quad 0<\max \left\{d_{i}\right\} K_{P}<1
$$

are satisfied.

The proof of Theorem 1 refers to [24], and thus is omitted here.

\section{Simulation Results}

In this section, the cyber-physical response to maintain the critical power supply using MG and emergency wireless communication is verified through the distributed power network detailed in Fig. 4, where two emergency MGs are available to maintain critical power supply. The MG including DG1 - DG4 is selected to evaluate the proposed centralised-to-distributed resilient control framework.

\section{A. Pre-event Wireless Communication Scheduling}

Considering the geographical location of DG1 - DG4 in Fig. 5, the efficient wireless network is formed as Fig. 4. The corresponding undirected graph $\mathcal{G}$ modelling distributed 


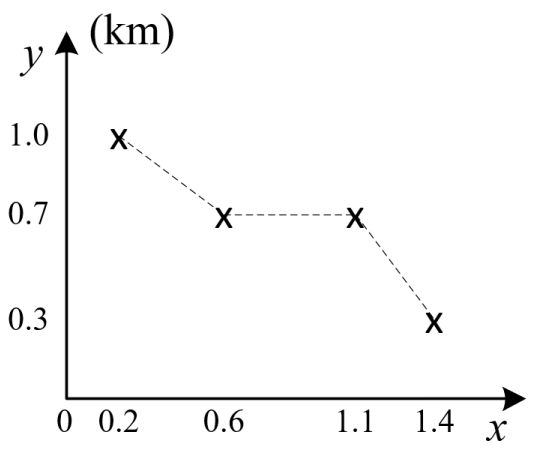

Fig. 5. Geographical location of DG1 - DG4.

TABLE II

PARAmeters of Pre-EVEnt C2C Wireless Network SCHEduling

\begin{tabular}{c|c}
\hline \hline Parameter & Value \\
\hline \hline sub-carrier bandwidth & $25 \mathrm{kHz}$ \\
\hline number of sub-carriers & 40 \\
\hline $\begin{array}{c}\text { maximum transmission power of } \\
\text { a controller wireless module }\end{array}$ & $24 \mathrm{dBm}$ \\
\hline $\begin{array}{c}\text { constant power of } \\
\text { a controller wireless module }\end{array}$ & $0.1 \mathrm{dBm}$ \\
\hline noise power & $-62 \mathrm{dBm}$ \\
\hline packet size & 32 bytes \\
\hline channel gain & $\begin{array}{c}g_{e, s}=h d_{e}^{-3} \\
\text { with } h=0.09\end{array}$ \\
\hline \hline
\end{tabular}

communication has the following characteristic:

$$
\mathcal{A}=\left[\begin{array}{llll}
0 & 1 & 0 & 0 \\
1 & 0 & 1 & 0 \\
0 & 1 & 0 & 1 \\
0 & 0 & 1 & 0
\end{array}\right], \mathcal{D}=\left[\begin{array}{llll}
1 & 0 & 0 & 0 \\
0 & 2 & 0 & 0 \\
0 & 0 & 2 & 0 \\
0 & 0 & 0 & 1
\end{array}\right]
$$

The parameters of the cyber layer are detailed in Table II [25], [26]. From the adjacent matrix $\mathcal{A}$, we can find there are six communication loops to be optimised. The optimised transmit data rate is $5.0878 \times 10^{3} \mathrm{bps}$, and the corresponding delay $\tau=50.3 \mathrm{~ms}$, thus the sampling interval in the controller of DGs is set as $T_{s}=100 \mathrm{~ms}$.

\section{B. Post-event Response Based on C2C Distributed Control}

The control performance of proposed $\mathrm{C} 2 \mathrm{C}$ distributed control is shown in Fig. 6. After the occurence of extreme conditions, such as earthquakes and hurricanes, the DGs maintain the critical power supply using primary control, which leads to the control deviation. Then, the secondary control is activated at $t=1 \mathrm{~s}$ with the parameters $K_{\omega}=1, K_{P}=0.4$, and the frequency deviation is reduced to zero. After that, the control objectives of Eqs. (3) and (4) can be reached, though the load demand changes. In order to verify the parameter design using Theorem 1 , we set $K_{\omega}=1, K_{P}=0.9$, and $K_{\omega}=2, K_{P}=0.4$, respectively. From Fig. 7 and Fig. 8, it is clear that the control performance declines in both scenarios. More specifically, $K_{P}=0.9$, exceeding to the boundary of

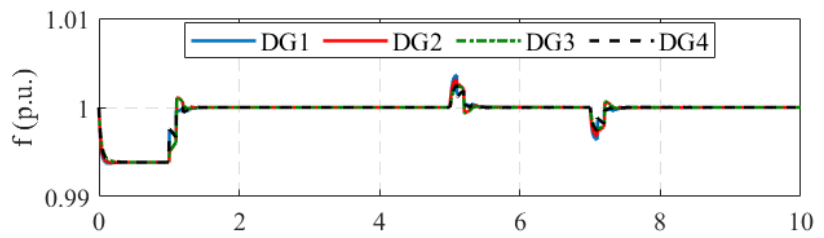

(a)

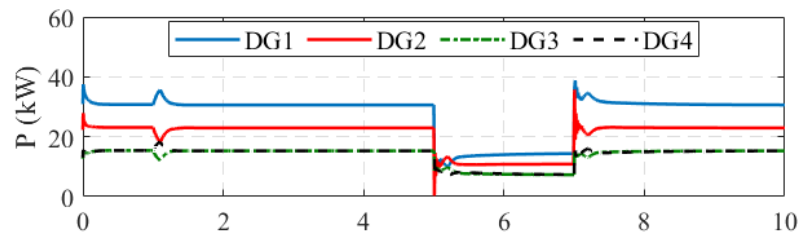

(b)

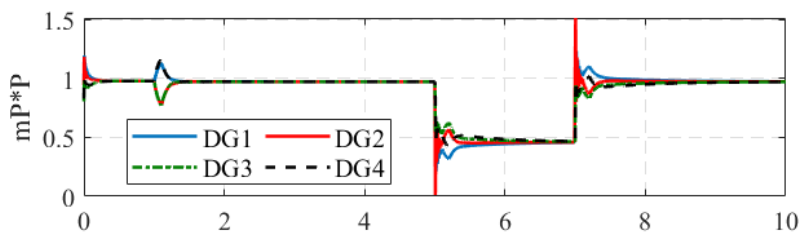

(c)

Time(sec)

Fig. 6. Control performance with rational $K_{\omega}, K_{P}$.

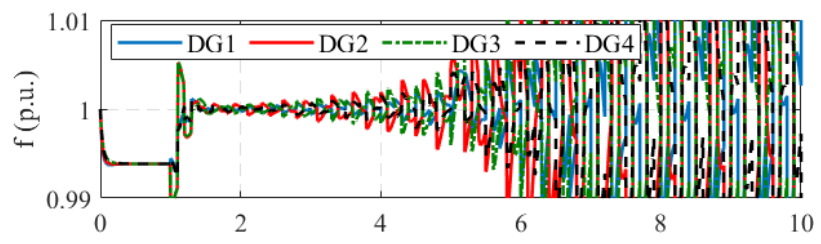

(a)

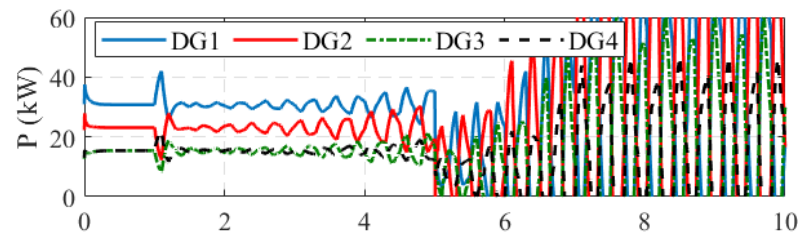

(b)

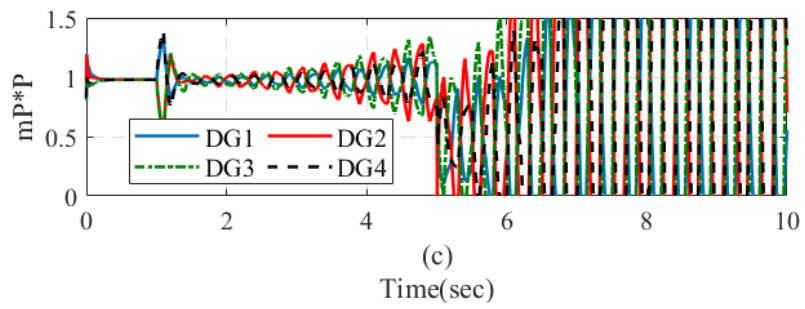

Fig. 7. Control performance with irrational $K_{P}$.

criteria Eq. (13), leads to the divergency in Fig. 7, while $K_{\omega}=2$ on the criteria boundary causes the oscillation of frequency regulation.

\section{CONClusion}

This paper proposes a centralised-to-distributed framework to enhance the resilience of power supply in response to extreme weather events. These failures have taught utilities, regu- 


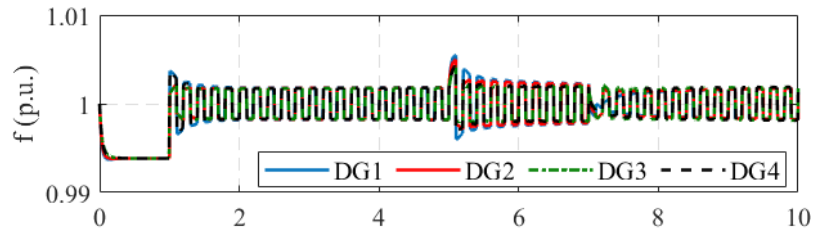

(a)

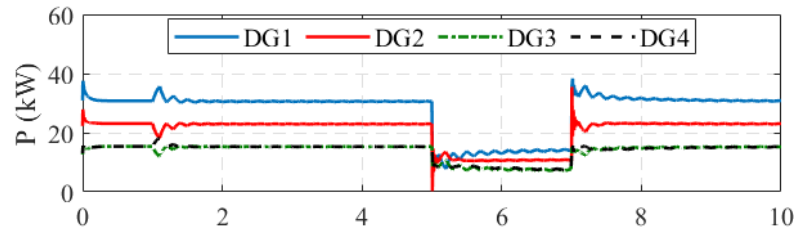

(b)

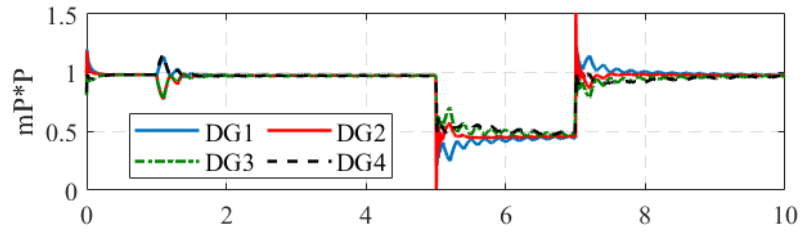

(c)

Time(sec)

Fig. 8. Control performance with irrational $K_{\omega}$.

lators, and stakeholders that faults cascade across the continental power grid, thus exacerbating a local weather phenomenon into a national, socioeconomic catastrophe. Traditional power systems are prone to such cascading power outages that last long periods of time and are complex and time-consuming to recover - in other words, not resilient. In the proposed resilient control framework, the cyber-physical response plan is dynamically updated in the centralised controller of distributed power network under the normal operation, in which cyber-layer $\mathrm{C} 2 \mathrm{C}$ distributed communication and physical-layer emergency MGs formation are scheduled. Considering the possible damage of base stations, the backup communication employs private wireless network that is specific for power industry to provide a reliable service for real-time control. The inevitable delay derived from the limited reliable bandwidth is then considered in the control system design. At last, the whole pre-event planning process and post-event response performance are evaluated through the presented case studies.

\section{REFERENCES}

[1] D. Apostolopoulou, S. Bahramirad, and A. Khodaei, "The interface of power: Moving toward distribution system operators," IEEE Power and Energy Magazine, vol. 14, no. 3, pp. 46-51, 2016.

[2] J. Wang and X. Lu, "Sustainable and resilient distribution systems with networked microgrids," Proceedings of the IEEE, vol. 108, no. 2, pp. 238-241, 2020.

[3] Y. Wang, C. Chen, J. Wang, and R. Baldick, "Research on resilience of power systems under natural disasters-a review," IEEE Transactions on Power Systems, vol. 31, no. 2, pp. 1604-1613, 2016.

[4] C. Konstantinou, "Towards a secure and resilient all-renewable energy grid for smart cities," IEEE Consumer Electronics Magazine, 2021.

[5] D. E. Olivares, A. Mehrizi-Sani, A. H. Etemadi, C. A. Cañizares, R. Iravani, M. Kazerani, A. H. Hajimiragha, O. Gomis-Bellmunt, M. Saeedifard, R. Palma-Behnke et al., "Trends in microgrid control," IEEE Transactions on smart grid, vol. 5, no. 4, pp. 1905-1919, 2014.
[6] R. Panora, J. E. Gehret, M. M. Furse, and R. H. Lasseter, "Real-world performance of a certs microgrid in manhattan," IEEE Transactions on Sustainable Energy, vol. 5, no. 4, pp. 1356-1360, 2014.

[7] C. Marnay, H. Aki, K. Hirose, A. Kwasinski, S. Ogura, and T. Shinji, "Japan's pivot to resilience: How two microgrids fared after the 2011 earthquake," IEEE Power and Energy Magazine, vol. 13, no. 3, pp. 4457, 2015.

[8] X. Wu, C. Shen, and R. Iravani, "A distributed, cooperative frequency and voltage control for microgrids," IEEE Transactions on Smart Grid, vol. 9, no. 4, pp. 2764-2776, 2018.

[9] P. Ge, B. Chen, and F. Teng, "Event-triggered distributed model predictive control for resilient voltage control of an islanded microgrid," International Journal of Robust and Nonlinear Control, vol. 31, no. 6, pp. 1979-2000, 2021.

[10] S. T. Cady, A. D. Domínguez-García, and C. N. Hadjicostis, "A distributed generation control architecture for islanded ac microgrids," IEEE Transactions on Control Systems Technology, vol. 23, no. 5, pp. 1717-1735, 2015.

[11] P. Ge, Y. Zhu, T. C. Green, and F. Teng, "Resilient secondary voltage control of islanded microgrids: An eskbf-based distributed fast terminal sliding mode control approach," IEEE Transactions on Power Systems, vol. 36, no. 2, pp. 1059-1070, 2021.

[12] R. H. Lasseter, Z. Chen, and D. Pattabiraman, "Grid-forming inverters: A critical asset for the power grid," IEEE Journal of Emerging and Selected Topics in Power Electronics, vol. 8, no. 2, pp. 925-935, 2020.

[13] H. M. Al-Suwaidan, S. Mohagheghi, and Q. Han, "A communication framework for an ad-hoc microgrid for disaster response," in 2015 IEEE International Conference on Smart Grid Communications (SmartGridComm). IEEE, 2015, pp. 834-839.

[14] Y. Du, X. Lu, J. Wang, B. Chen, H. Tu, and S. Lukic, "Dynamic microgrids in resilient distribution systems with reconfigurable cyberphysical networks," IEEE Journal of Emerging and Selected Topics in Power Electronics, 2020.

[15] W. Shao, Z. Ling, J. Li, Y. Lu, W. Bai, and D. Zhang, "Evolution trend of lte $230 \mathrm{mhz}$ electric wireless private network technology," in 2019 IEEE International Conference on Energy Internet (ICEI). IEEE, 2019, pp. 591-595.

[16] M. Higgins, F. Teng, and T. Parisini, "Stealthy mtd against unsupervised learning-based blind fdi attacks in power systems," IEEE Transactions on Information Forensics and Security, vol. 16, pp. 1275-1287, 2021.

[17] J. W. Simpson-Porco, Q. Shafiee, F. Dörfler, J. C. Vasquez, J. M. Guerrero, and F. Bullo, "Secondary frequency and voltage control of islanded microgrids via distributed averaging," IEEE Transactions on Industrial Electronics, vol. 62, no. 11, pp. 7025-7038, 2015.

[18] H. Zhang, S. Kim, Q. Sun, and J. Zhou, "Distributed adaptive virtual impedance control for accurate reactive power sharing based on consensus control in microgrids," IEEE Transactions on Smart Grid, vol. 8, no. 4, pp. 1749-1761, 2017.

[19] A. Bidram, A. Davoudi, and F. L. Lewis, "A multiobjective distributed control framework for islanded ac microgrids," IEEE Transactions on industrial informatics, vol. 10, no. 3, pp. 1785-1798, 2014.

[20] P. Ge, X. Dou, X. Quan, Q. Hu, W. Sheng, Z. Wu, and W. Gu, "Extended-state-observer-based distributed robust secondary voltage and frequency control for an autonomous microgrid," IEEE Transactions on Sustainable Energy, vol. 11, no. 1, pp. 195-205, 2020.

[21] A. Goldsmith, Wireless communications. Cambridge university press, 2005.

[22] J. Lofberg, "Yalmip: A toolbox for modeling and optimization in matlab," in 2004 IEEE international conference on robotics and automation (IEEE Cat. No. 04CH37508). IEEE, 2004, pp. 284-289.

[23] Mosek ApS. [Online]. Available: www.mosek.com

[24] Y.-P. Tian and C.-L. Liu, "Consensus of multi-agent systems with diverse input and communication delays," IEEE Transactions on Automatic Control, vol. 53, no. 9, pp. 2122-2128, 2008.

[25] P.-Y. Kong, "Multicell d2d communications for hierarchical control of microgrid system," IEEE Systems Journal, 2020.

[26] A. Termehchi and M. Rasti, "Joint sampling time and resource allocation for power efficiency in industrial cyber-physical systems," IEEE Transactions on Industrial Informatics, vol. 17, no. 4, pp. 2600-2610, 2021. 